\title{
Impact of obesity on the increasing incidence of type 1 diabetes
}

\section{Raffaella Buzzetti MD ${ }^{1} \quad$ Simona Zampetti PhD ${ }^{1} \quad$ Paolo Pozzilli MD ${ }^{2,3}$ (c)}

${ }^{1}$ Department of Experimental Medicine, Sapienza University, Rome, Italy

${ }^{2}$ Department of Medicine, Unit of Endocrinology and Diabetes, Campus BioMedico University of Rome, Rome, Italy

${ }^{3}$ Centre of Immunobiology, Barts and London School of Medicine and Dentistry, Queen Mary University of London, London, UK

\section{Correspondence}

Professor Paolo Pozzilli, MD, Department of Medicine, Unit of Endocrinology and Diabetes, Campus Bio-Medico University of Rome, Via Alvaro del Portillo 21, 00128 Rome, Italy. Email: p.pozzilli@unicampus.it

Peer Review

The peer review history for this article is available at https://publons.com/publon/10. 1111/dom.14022.

\begin{abstract}
Published estimates of the incidence of type 1 diabetes (T1D) in children in the last decade varies between $2 \%$ and $4 \%$ per annum. If this trend continued, the disease incidence would double in the next 20 years. The risk of developing T1D is determined by a complex interaction between multiple genes (mainly human leukocyte antigens) and environmental factors. Notwithstanding that genetic susceptibility represents a relevant element in T1D risk, genetics alone cannot explain the increase in incidence. Various environmental factors have been suggested as potential triggers for T1D, including several viruses and the hygiene hypothesis; however, none of these seems to explain the large increase in T1D incidence observed over the last decades. Several studies have demonstrated that the prevalence of childhood/adolescence overweight and obesity has risen during the past 30 years in T1D. Currently, at diagnosis, the majority of patients with T1D have normal or elevated body weight and $~ 50 \%$ of patients with longstanding T1D are either overweight or obese. The growing prevalence of obesity in childhood and adolescence offers a plausible explanation for the increase in T1D incidence observed in recent decades. Possible mechanisms of the enhancement of $\beta$-cell autoimmunity by obesity include: a) insulin resistance-induced $\beta$-cell secretory demand triggering autoimmunity through cytokine release, neo-epitope antigen formation and increase in $\beta$-cell apoptosis, and b) obesity-induced low-grade inflammation with pro-inflammatory cytokines secreted by locally infiltrating macrophages, which contribute to the presentation by islet cells of autoantigens generally not accessible to $\mathrm{T}$ cells. Further studies are needed to clarify whether the control of body weight can prevent or delay the current and continuing rise in T1D incidence.
\end{abstract}

\section{KEYWORDS}

type 1 diabetes, beta cell function, glycaemic control

\section{1 | INCIDENCE OF TYPE 1 DIABETES IS ON THE INCREASE}

Type 1 diabetes (T1D) represents $\sim 10 \%$ of all cases of diabetes mellitus. ${ }^{1}$ T1D is recognized to be an autoimmune-mediated disease leading to the destruction of insulin-producing $\beta$ cells. ${ }^{1,2}$ This disease occurs mostly during childhood/adolescence, and there has been an increase in the incidence of the disease in early life. ${ }^{3}$ However, nearly half of patients experience onset in adulthood. ${ }^{4}$
DIAMOND 5 and EURODIAB, ${ }^{6}$ two large epidemiological studies evaluating the incidence and trends in T1D diabetes worldwide and in Europe, respectively, have highlighted the increasing incidence of this disease. Specifically, in the period 1990 to 2000 in children aged 0 to 14 years, the estimated annual increase in incidence worldwide was $2.8 \%$ per annum and in Europe it was 3.9\% per annum. More recently, Patterson et al ${ }^{7}$ analysed the age-/sex-standardized incidence rates for the age group 0 to 14 years in 26 European centres registering newly diagnosed individuals in geographically defined regions for up 
to 25 years during the period 1989 to 2013 . A significant increase in incidence was noted in all centres, but not in two high-incidence countries (Finland and Norway), where reducing increase rates were observed in recent years. ${ }^{7}$ This is an interesting finding because it was associated with a plateau in the growth of obesity in these countries. ${ }^{8}$ Despite these findings, a pooled analysis across all European centres revealed that the incidence of childhood T1D continues to rise by $\sim 3 \%$ to $4 \%$ per annum. ${ }^{7}$ If this trend continued, the incidence would double in the next 20 years. To support the relevance of the role of obesity in the growing incidence of T1D, the reported increased incidence of T1D in Kuwait which parallels the rise of obesity over the past quarter of century is of particular interest. ${ }^{9}$

A very recent study, performed in French children, reported that in a total of 12067 children identified as newly diagnosed with T1D, the annual incidence rate increased from 15.4 to 19.1 per 100000 person/year between 2010 and $2015{ }^{10}$ The increase in the annual rate was $4.0 \%$. In the United States, pooled data from five centres on children and adolescents diagnosed before the age of 20 years indicated a 1.8\% (95\% Cl 1.0, 2.6) annual increase during 2002 to 2012 after adjustment for age, gender and race or ethnic group. ${ }^{11}$

Regarding the Asian population, a study performed in Zhejiang province, in a region of China with low T1D incidence, showed that the age-standardized T1D incidence in children and adolescents aged 0 to 19 years doubled between 2007 (1.22/100 000 person-years) and 2013 (2.48/100 000 person-years), with an average annual increase of $12 \%{ }^{12}$ In a retrospective cohort study in South Korea conducted for the period 2001 to 2010, the average incidence rate of T1D in children aged $<15$ years was 2.01/100 000. ${ }^{13}$ The reported incidence of T1D in Korean children and adolescents from 2012 to 2014 increased to $3.19 / 100000 .^{14}$

In Japan, the incidence of childhood-onset T1D for 2005 to 2010 was $2.25 / 100000 .{ }^{15}$ The incidence was not only shown to be markedly lower than in Western countries, but has also remained unchanged for $>10$ years, with no evidence of T1D spreading to younger age groups. ${ }^{15}$

Finally, some reports suggest a temporal increase in incidence trends not only in children (0-14 years) but also in adolescents and young adults (15-30 years), suggesting an increasing effect over time of environmental factors. ${ }^{16-19}$

\section{2 | INTERACTION BETWEEN GENETICS AND ENVIRONMENT IN THE INCREASING INCIDENCE OF TYPE 1 DIABETES}

The risk of developing T1D is determined by a complex interaction between multiple genes and environmental factors. Evidence for a genetic component is provided by the higher risk in siblings of people with T1D (6\%) compared to that observed in the general population ( 0.4\%), and there are at least 20 regions of the genome predisposing to $\mathrm{T} 1 \mathrm{D} .{ }^{20}$ The human leukocyte antigen (HLA) region accounts for $\sim 50 \%$ of the genetic susceptibility to T1D; the significant role played by the HLA system in susceptibility to T1D could affect both the incidence and age of onset of the disease. ${ }^{21,22}$ Indeed, the minor frequency of HLA class II high-risk genotypes can partially explain the low incidence of T1D reported in central Italy. ${ }^{23}$

Notwithstanding that genetics represents a relevant element in T1D risk, the increase in T1D incidence observed worldwide in recent decades cannot be explained by genetic susceptibility. Moreover, it is worth noting that the rising incidence of T1D has been accompanied by an increase of the disease in patients carrying low/moderate HLA risk genotypes compared to previous decades. ${ }^{24}$

These observations underline the less important role for genetic predisposition in the increasing incidence of T1D, and the same time highlight the role played by a number of diverse environmental factors in the rapid change in the global incidence of T1D. ${ }^{25}$

It has long been suggested that viral infections could be environmental triggers implicated in the aetiology of T1D. Enteroviruses, coxsackie virus B, cytomegalovirus, rotaviruses and encephalomyocarditis virus might contribute to T1D pathogenesis. ${ }^{25,26}$ Alternatively, according to the hygiene hypothesis, put forward to explain the rise in immune dysregulation, the increase in T1D could be explained by the decrease in bacterial and viral infections, which could indeed have served as a defence mechanism against developing the disease. ${ }^{27}$ Recent evidence indicates that there has been a decrease in global regional and national morbidity by $30 \%$ and in mortality by $51 \%$ with regard to respiratory infections in children, probably as a result of increases in vaccinations and other prevention measures. ${ }^{28-30}$ Nonetheless, the identification and confirmation of environmental determinants remain a formidable challenge. ${ }^{31}$ Because of the lack of strong evidence for a single factor as a major trigger for T1D, it is possible that numerous elements may determine additive or synergistic effects by acting via several mechanisms and/or at different stages in the disease process. ${ }^{32}$ Environmental exposures may occur at any time before the onset of the disease from in utero to T1D onset. ${ }^{33}$ In addition, environmental determinants may vary in different populations, also depending on the genetic background.

To summarize, various different causal factors have been proposed as triggers for T1D; however, none of these elements seems to explain the increase in incidence of T1D observed in the past decades. The identification of environmental determinants contributing to T1D has been difficult because of the heterogeneous and transitory nature of environmental exposures.

\section{3 | THE OBESOGENIC ENVIRONMENT AND HOW IT MAY LEAD TO T1D}

Numerous studies have now demonstrated that the prevalence of childhood overweight and obesity has risen during the past 30 years in children with T1D since diagnosis. ${ }^{34,35}$ Obesity per se could be linked to the increasing incidence of T1D, generating a novel expression of the disease known as "double diabetes". ${ }^{36}$ The worldwide prevalence of obesity increased from $<1 \%$ in 1975 to $7.8 \%$ and to $5.6 \%$ in 2016 for boys and girls, respectively. ${ }^{37}$ This trend increases similarly or even at a faster rate in patients with T1D compared to the 
general population. ${ }^{38}$ Nowadays, a good number of T1D patients at diagnosis, especially adults but also children, have a normal or slightly raised body mass index (BMI). ${ }^{39}$ A study conducted in $~ 9000$ children with T1D demonstrated that higher BMI was associated with younger age at onset, concluding that the elevated weight gain could be a risk factor for an early manifestation of the disease. ${ }^{39}$ More recently, it has been demonstrated that children with overweight/obesity have a $63 \%$ higher risk of developing T1D. ${ }^{40}$ All these new observations support the "accelerator hypothesis" put forward by Wilkin in 2001 . He suggested a link between BMI and T1D, ${ }^{41}$ underlining that T1D and type 2 diabetes (T2D) are the same disorder of insulin resistance set against different genetic background. T1D and T2D in terms of pathogenesis represent a continuum as the interaction between genetic susceptibility and insulin resistance determines an earlier onset of T1D in obese compared to non-obese subjects. Obesity favours insulin resistance, causing stress on $\beta$ cells and rendering them more vulnerable to an autoimmune attack. ${ }^{42}$ The Wilkin's hypothesis received definitive confirmation by a recent study testing whether raised $\mathrm{BMI}$ accelerates islet autoimmunity before T1D diagnosis. The rate of progression from single to multiple autoantibodies increased in those with high BMI, particularly in children/adolescents with moderate/low HLA risk. ${ }^{43}$

With regard to the mechanisms involved in obesity-induced $\beta$-cell damage, several mechanisms may be considered to explain the activation of an autoimmune response in obese subjects (Table 1). Although there is no direct causal link between obesity and the increased incidence of T1D, in the presence of insulin resistance, $\beta$ cells compensate by secreting more insulin. Such increased demand leads to an imbalance with respect to the ability of the islet cell endoplasmic reticulum to produce insulin, leading to $\beta$-cell stress with consequent cytokine release [interferon (IFN)a], neo-epitope formation and increase in $\beta$-cell apoptosis. ${ }^{44,45}$ IFNa may induce and amplify the adaptive immune response against human $\beta$ cells, thus having a central role in the early phases of T1D. ${ }^{44}$

TABLE 1 Possible mechanisms underlying how obesity may lead to enhanced $\beta$-cell specific autoimmunity and destruction in type 1 diabetes

- Obesity can accelerate the clinical onset of autoimmunity by increasing insulin resistance and thus insulin requirement in genetically susceptible subjects

- Obesity, overfeeding and insulin resistance increase $\beta$-cell secretory demand and lead to the activation of intrinsic $\beta$-cell stress, triggering autoimmunity through: (a) cytokine release (IFNa), neo-epitope formation and increase in $\beta$-cell apoptosis and (b) acceleration of autoimmune mediated $\beta$-cell death

- Obesity-induced low-grade inflammation (secretion by stressed adipocytes undergoing apoptosis of pro-inflammatory cytokines, such as IL1- $\beta$, IL6, TNF alpha) contributing to: (a) release from islet cells of auto-antigens generally not accessible to $T$ cells and (b) $\beta$-cell death

Abbreviations: IFN, interferon; IL, interleukin.
Another possible mechanism is the obesity-induced low-grade inflammation at the core of the $\beta$-cell damaging process. ${ }^{46}$ Obesity and insulin resistance lead to promotion of hepatic de novo lipogenesis, initial liver steatosis, non-alcoholic fatty liver disease, nonalcoholic steatohepatitis, pancreatic fat accumulation and, most important, tissue macrophage infiltration. ${ }^{47}$ Low-grade systemic inflammation develops from adipose tissue dysfunction, underlined by the presence of infiltrating macrophages. Adipocytes undergo apoptosis and release antigens previously not available to the immune system. ${ }^{45}$ Macrophages synthesize and secrete a variety of chemokines capable of recruiting more lymphocytes into pancreatic tissue. ${ }^{48,49}$ The presence of chemokines, markedly enhanced by $\beta$-cell exposure to inflammatory signals, ${ }^{50,51}$ induces two specific chemokine receptors (i.e. CXCL1-2 and CXCR3) $)^{52,53}$ which may contribute to the expansion of the already ongoing autoimmune activation. We demonstrated that the frequency of autoantibodies to islet antigen-2 (IA-

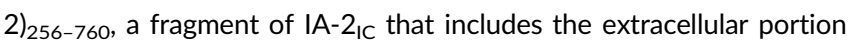
of the protein, increased with increasing $B M I$ in patients with latent autoimmune diabetes in adults. ${ }^{54}$ We have also found that IA2 256-760 autoantibodies have a higher frequency in obese subjects with normal glucose tolerance compared to subjects with T2D. ${ }^{55}$ Thus, we suggest that an increasing "obesogenic" environment and the consequent associated low-grade inflammation could promote/ amplify, in obese subjects, the development of diabetes-specific autoimmunity. ${ }^{56}$

\section{4 | IMPLICATIONS FOR OVERWEIGHT/ OBESITY AT T1D DIAGNOSIS}

The observation that overweight/obesity can characterize T1D at clinical diagnosis or even in the prediabetic state, drives us to reconsider the rationale for intervention trials. ${ }^{57}$ Such trials focus mainly on targeting the autoimmune response to $\beta$ cells, with a number of compounds acting at different stages of the immunological events associated with $\beta$-cell destruction. ${ }^{58}$ Since autoimmunity continues for at least few years after diagnosis, increasing the loss of residual $\beta$-cell function, interventions to lessen this phenomenon with drugs targeting obesity-driven systemic inflammation may slow down the progression of islet cell destruction. Recent trials have been conducted with this aim. ${ }^{59}$ Further studies are needed to clarify whether the control of body weight can prevent or delay the current and continuing rise in T1D incidence. Furthermore, in patients with longstanding T1D, overweight/obesity may be the result of the general anabolic effect caused by iatrogenic insulin replacement. The perceived threat of hypoglycaemia, which leads to a significant increase in calorie intake, is another factor causing body weight increase. In this respect, glucagon-like peptide-1 receptor agonists have been proposed as additional therapy in obese patients with pump-treated T1D. ${ }^{60}$

In conclusion, the clinical phenotype of T1D is dramatically changing compared to the past, and clinicians should be aware of this modification as it has both diagnostic and clinical implications. 


\section{CONFLICTS OF INTEREST}

R.B. has served on advisory boards for Sanofi and Abbott, and has received honoraria for speakers' bureau and travel reimbursements from Sanofi, Eli Lilly, AstraZeneca, Novo Nordisk, Abbott and MSD. S.Z. declares no conflict of interest. P.P. participated in advisory boards for Sanofi and AstraZeneca, and has received honoraria and travel reimbursements from Sanofi, Eli Lilly, AstraZeneca, Medtronic, Abbott and MSD.

\section{AUTHOR CONTRIBUTIONS}

All authors have contributed equally to: generation of concepts, reviewing data in the literature, writing the manuscript and agreeing on the final draft of the paper.

\section{ORCID}

Paolo Pozzilli (DD https://orcid.org/0000-0001-5090-636X

\section{REFERENCES}

1. Atkinson MA, Eisenbarth GS, Michels AW. Type 1 diabetes. Lancet. 2014;383:69-82

2. Incani M, Serafini C, Satta C, et al. High prevalence of diabetesspecific autoimmunity in first-degree relatives of Sardinian patients with type 1 diabetes. Diabetes Metab Res Rev. 2017;33(3):e2864. https://doi.org/10.1002/dmrr.2864.

3. You WP, Henneberg M. Type 1 diabetes prevalence increasing globally and regionally: the role of natural selection and life expectancy at birth. BMJ Open Diabetes Res Care. 2016;4:1-7.

4. Monaghan $M$, Helgeson V, Wiebe D. Type 1 diabetes in young adulthood. Curr Diabetes Rev. 2015;11:239-250.

5. The DIAMOND Project Group. Incidence and trends of childhood type 1 diabetes worldwide 1990-1999. Diabet Med. 2006;23: 857-866.

6. Patterson CC, Dahlquist GG, Gyürüs E, Green A, Soltész G, The EURODIAB Study Group. Incidence trends for childhood type 1 diabetes in Europe during 1989-2003 and predicted new cases 2005-20: a multicentre prospective registration study. Lancet. 2009;373:20272033.

7. Patterson CC, Harjutsalo V, Rosenbauer J, Neu A, et al. Trends and cyclical variation in the incidence of childhood type 1 diabetes in 26 European centres in the 25year period 1989-2013: a multicentre prospective registration study. Diabetologia. 2019;62:408-417.

8. Public Health Report. Overweight and obesity in Norway. https:// www.fhi.no/en/op/hin/lifestyle/overweight-and-obesity-innorway-/. Published March 10, 2011. Updated November 3, 2017. Accessed February 17, 2020.

9. Shaltout AA, Wake D, Thanaraj TA, et al. Incidence of type 1 diabetes has doubled in Kuwaiti children 0-14 years over the last 20 years. Pediatr Diabetes. 2017;18:761-766.

10. Piffaretti C, Mandereau-Bruno L, Guilmin-Crepon S, Choleau C, Coutant R, Fosse-Edorh S. Trends in childhood type 1 diabetes incidence in France, 2010-2015. Diabetes Res Clin Pract. 2019;149: 200-207.

11. Mayer-Davis EJ, Lawrence JM, Dabelea D, et al. Incidence trends of type 1 and type 2 diabetes among youths, 2002-2012. N Engl J Med. 2017;376:1419-1429.

12. Wu HB, Zhong JM, Hu RY, et al. Rapidly rising incidence of type 1 diabetes in children and adolescents aged 019 years in Zhejiang, China, 2007 to 2013. Diabet Med. 2016;33:1339-1346.

13. Lee JH, Kim YM, Kwak MJ, et al. Incidence trends and associated factors of diabetes mellitus in Korean children and adolescents: a retrospective cohort study in Busan and Gyeongnam. Ann Pediatr Endocrinol Metab. 2015;20:206-212.

14. Kim JH, Lee CG, Lee YA, Yang SW, Shin CH. Increasing incidence of type 1 diabetes among Korean children and adolescents: analysis of data from a nationwide registry in Korea. Pediatr Diabetes. 2016;17: 519-524.

15. Onda $\mathrm{Y}$, Sugihara $\mathrm{S}$, Ogata $\mathrm{T}$, et al. Incidence and prevalence of childhood-onset type 1 diabetes in Japan: the T1D study. Diabet Med. 2017;34:909-915.

16. Bruno G, Merletti F, Biggeri A, et al. Increasing trend of type I diabetes in children and young adults in the province of Turin (Italy). Analysis of age, period and birth cohort effects from 1984 to 1996. Diabetologia. 2001;44:22-25.

17. Fröhlich-Reiterer EE, Holl RW, et al. Predictors of increasing BMI during the course of diabetes in children and adolescents with type 1 diabetes: data from the German/Austrian DPV multicentre survey. Arch Dis Child. 2014;99:738-743.

18. Schwandt A, Holl RW, et al. Longitudinal trajectories of metabolic control from childhood to young adulthood in type 1 diabetes from a large German/Austrian registry: a group-based Modeling approach. Diabetes Care. 2017:40:309-316.

19. DuBose SN, Hermann JM, Tamborlane WV, et al. Type 1 diabetes exchange clinic network and diabetes prospective follow-up registry. Obesity in youth with type 1 diabetes in Germany, Austria, and the United States. J Pediatr. 2015;167:627-632.

20. Steck AK, Rewers MJ. Genetics of type 1 diabetes. Clin Chem. 2011; 57:176-185.

21. Mishra R, Åkerlund $\mathrm{M}$, Cousminer $\mathrm{DL}$, et al. Genetic discrimination between LADA and childhood-onset type 1 diabetes within the MHC. Diabetes Care. 2020;43:418-425. https://doi.org/10.2337/ dc19-0986.

22. Buzzetti R, Prudente S, Copetti M, et al. Clinical worthlessness of genetic prediction of common forms of diabetes mellitus and related chronic complications: a position statement of the Italian Society of Diabetology. Nutr Metab Cardiovasc Dis. 2017;27:99-114.

23. Petrone A, Bugawan TL, Mesturino CA, et al. The distribution of HLA class II susceptible/protective haplotypes could partially explain the low incidence of type 1 diabetes in continental Italy (Lazio region). Tissue Antigens. 2001;58:385-394.

24. Vehik K, Hamman RF, Lezotte D, et al. Trends in high-risk HLA susceptibility genes among Colorado youth with type 1 diabetes. Diabetes Care. 2008;31:1392-1396.

25. Rewers M, Ludvigsson J. Environmental risk factors for type 1 diabetes. Lancet. 2016;387:2340-2348.

26. Nekoua MP, Bertin A, Sane F, et al. Pancreatic beta cells persistently infected with coxsackievirus B4 are targets of NK cell-mediated cytolytic activity. Cell Mol Life Sci. 2020;77:179-194. https://doi.org/10. 1007/s00018-019-03168-4 Epub 2019 Jun 6.

27. Jakobsen OAJ, Szereday L. The "three amigos" lurking behind type 1 diabetes: hygiene, gut microbiota and viruses. Acta Microbiol Immunol Hung. 2018;65:421-438.

28. GBD 2016 Lower Respiratory Infections Collaborators. Estimates of the global, regional, and national morbidity, mortality, and aetiologies of lower respiratory infections in 195 countries, 1990-2016: a systematic analysis for the global burden of disease study 2016. Lancet Infect Dis. 2018;18:1191-1210.

29. McAllister DA, Liu L, Shi T, et al. Global, regional, and national estimates of pneumonia morbidity and mortality in children younger than 5 years between 2000 and 2015: a systematic analysis. Lancet Glob Health. 2019;7:47-57.

30. Wang $\mathrm{X}, \mathrm{Li} \mathrm{Y}, \mathrm{O}$ 'Brien $\mathrm{KL}$, et al. Global burden of respiratory infections associated with seasonal influenza in children under 5 years in 2018: a systematic review and modelling study. Lancet Glob Health. 2020. https://doi.org/10.1016/S2214-109X(19)30545-5. 
31. Knip M, Veijola R, Virtanen SM, Hyöty H, Vaarala O, Åkerblom HK. Environmental triggers and determinants of type 1 diabetes. Diabetes. 2005;54:S125-S136.

32. Rønningen KS. Environmental trigger(s) of type 1 diabetes: why so difficult to identify? Biomed Res Int. 2015. https://doi.org/10.1155/ 2015/321656.

33. Hagopian WA, Erlich $\mathrm{H}$, Lernmark $\mathrm{A}$, et al. The environmental determinants of diabetes in the young (TEDDY): genetic criteria and international diabetes risk screening of 421000 infants. Pediatr Diabetes. 2011;12:733-743.

34. De Keukelaere M, Fieuws S, Reynaert N, et al. Evolution of body mass index in children with type 1 diabetes mellitus. Eur J Pediatr. 2018;177:1661-1666.

35. Corbin KD, Driscoll KA, Pratley RE, et al. Obesity in type 1 diabetes: pathophysiology, clinical impact, and mechanisms. Endocr Rev. 2018; 39:629-663.

36. Pozzilli P, Buzzetti R. A new expression of diabetes: double diabetes. Trends Endocrinol Metab. 2007;18:52-57.

37. Worldwide Trends in Body-Mass Index. Underweight, overweight, and obesity from 1975 to 2016: a pooled analysis of 2416 population-based measurement studies in 128.9 million children, adolescents, and adults. Lancet. 2017;390:2627-2642.

38. Fellinger $\mathrm{P}$, Fuchs $\mathrm{D}$, Wolf $\mathrm{P}$, et al. Overweight and obesity in type 1 diabetes equal those of the general population. Wien Klin Wochenschr. 2019;131:55-60.

39. Knerr I, Wolf J, Reinehr T, et al. The 'accelerator hypothesis': relationship between weight, height, body mass index and age at diagnosis in a large cohort of 9,248 German and Austrian children with type 1 diabetes mellitus. Diabetologia. 2005;48:2501-2504.

40. Ferrara CT, Geyer SM, Liu YF, et al. Excess BMI in childhood: a modifiable risk factor for type 1 diabetes development? Diabetes Care. 2017;40:698-701.

41. Wilkin TJ. The accelerator hypothesis: weight gain as the missing link between type I and type II diabetes. Diabetologia. 2001;44:914-922.

42. Saltiel AR, Olefsky JM. Inflammatory mechanisms linking obesity and metabolic disease. J Clin Invest. 2017;127:1-4.

43. Ferrara-Cook C, Geyer SM, Evans-Molina C, et al. Excess BMI accelerates islet autoimmunity in older children and adolescents. Diabetes Care. 2020;43:580-517.

44. Marroqui L, Dos Santos RS, Op de Beeck A, et al. Interferon-a mediates human beta cell HLA class I overexpression, endoplasmic reticulum stress and apoptosis, three hallmarks of early human type 1 diabetes. Diabetologia. 2017;60:656-667.

45. Brooks-Worrell BM, Palmer JP. Setting the stage for islet autoimmunity in type 2 diabetes: obesity-associated chronic systemic inflammation and endoplasmic reticulum (ER) stress. Diabetes Care. 2019;42: 2338-2346.
46. Versini M, Jeandel PY, Rosenthal E, Shoenfeld Y. Obesity in autoimmune diseases: not a passive bystander. Autoimmun Rev. 2014;13:981-1000.

47. Roden M, Shulman GI. The integrative biology of type 2 diabetes. Nature. 2019;576:51-60.

48. Sarkar SA, Lee CE, Victorino $F$, et al. Expression and regulation of chemokines in murine and human type 1 diabetes. Diabetes. 2012;61: 436-446.

49. Collier JJ, Sparer TE, Karlstad MD, Burke SJ. Pancreatic islet inflammation: an emerging role for chemokines. J Mol Endocrinol. 2017;59: 33-46.

50. Burke SJ, Collier JJ. Transcriptional regulation of chemokine genes: a link to pancreatic islet inflammation? Biomolecules. 2015;26:10201034.

51. Lopes M, Kutlu B, Miani M, et al. Temporal profiling of cytokineinduced genes in pancreatic $\beta$-cells by meta-analysis and network inference. Genomics. 2014;103:264-275.

52. Burke SJ, Lu D, Sparer TE, et al. NF-kB and STAT1 control CXCL1 and CXCL2 gene transcription. Am J Physiol Endocrinol Metab. 2014; 306:131-149.

53. Burke SJ, Karlstad MD, Eder AE, et al. Pancreatic $\beta$-cell production of CXCR3 ligands precedes diabetes onset. Biofactors. 2016;42: 703-715.

54. Buzzetti R, Spoletini M, Zampetti S, et al. Tyrosine phosphatase-related islet antigen 2(256-760) autoantibodies, the only marker of islet autoimmunity that increases by increasing the degree of BMI in obese subjects with type 2 diabetes. Diabetes Care. 2015;38:513-520.

55. Tiberti C, Zampetti S, Capoccia D, et al. Evidence of diabetes-specific autoimmunity in obese subjects with normal glucose tolerance. Diabetes Metab Res Rev. 2018 Nov;34(8):e3055.

56. Buzzetti R, Zampetti S, Maddaloni E. Adult-onset autoimmune diabetes: current knowledge and implications for management. Nat Rev Endocrinol. 2017;13:674-686.

57. Pozzilli P, Maddaloni E, Buzzetti R. Combination immunotherapies for type 1 diabetes mellitus. Nat Rev Endocrinol. 2015;11:289-297.

58. Warshauer JT, Bluestone JA, Anderson MS. New frontiers in the treatment of type 1 diabetes. Cell Metab. 2020;31:46-61.

59. Cobo-Vuilleumier N, Gauthier BR. Time for a paradigm shift in treating type 1 diabetes mellitus: coupling inflammation to islet regeneration. Metabolism. 2020;104:154137. https://doi.org/10. 1016/j.metabol.2020.154137.

60. Dejgaard TF, Schmidt S, Frandsen CS, et al. Liraglutide reduces hyperglycaemia and body weight in overweight, dysregulated insulin-pump-treated patients with type 1 diabetes: the lira pump trial-a randomized, double-blinded, placebo-controlled trial. Diabetes Obes Metab. 2019;22:492-500. https://doi.org/10.1111/dom. 13911. 